\title{
Achievable ADC Performance by Postcorrection Utilizing Dynamic Modeling of the Integral Nonlinearity
}

\author{
Niclas Björsell' ${ }^{1}$ and Peter Händel ${ }^{2}$ \\ ${ }^{1}$ ITB/Electronics, University of Gävle, 80176 Gävle, Sweden \\ ${ }^{2}$ Signal Processing Lab., ACCESS Linnaeus Center, School of Electrical Engineering, Royal Institute of Technology, \\ 10044 Stockholm, Sweden
}

Correspondence should be addressed to Niclas Björsell, niclas.bjorsell@hig.se

Received 1 May 2007; Revised 24 September 2007; Accepted 19 December 2007

Recommended by Boris Murmann

\begin{abstract}
There is a need for a universal dynamic model of analog-to-digital converters (ADC's) aimed for postcorrection. However, it is complicated to fully describe the properties of an ADC by a single model. An alternative is to split up the ADC model in different components, where each component has unique properties. In this paper, a model based on three components is used, and a performance analysis for each component is presented. Each component can be postcorrected individually and by the method that best suits the application. The purpose of postcorrection of an ADC is to improve the performance. Hence, for each component, expressions for the potential improvement have been developed. The measures of performance are total harmonic distortion (THD) and signal to noise and distortion (SINAD), and to some extent spurious-free dynamic range (SFDR).
\end{abstract}

Copyright (c) 2008 N. Björsell and P. Händel. This is an open access article distributed under the Creative Commons Attribution License, which permits unrestricted use, distribution, and reproduction in any medium, provided the original work is properly cited.

\section{INTRODUCTION}

The analog to digital converter (ADC) is a key component in many applications, for example, radio base stations and test and measurement instruments. In state-of-the-art designed vector signal analyzers (VSAs), the ADC is the bottle neck and an improvement in ADC performance directly improves the VSA performance. Characterization and testing of ADC's are important in many different aspects. One example is ADC postcorrection, where improvements in the ADC characteristics are obtained by digital signal processing methods, in particular the error occurrence is predicted in order to compensate for error source effects. A survey of error compensation methods is given in [1]. Postcorrection is built on the model of the ADC. A survey of state-of-theart ADC modeling techniques and models may be found in [2]. Normally, the ADC model is based on a characterization performed in high-performance test setups, whereupon an off-chip postcorrection algorithm is developed. In the literature, a majority of the proposed ADC characterization methods describes the static properties of the converter. A common solution for postcorrection based on a static model is the use of lookup tables (LUTs); that is, the ADC output is remapped using a table lookup, where the table entries are such that some performance measure is improved, as for example [3]. ADC postcorrection by table lookup methods has shown to improve performance measures such as spurious free dynamic range (SFDR), total harmonic distortion (THD), and signal-to-noise and distortion ratio (SINAD). It has been shown that postcorrection based on LUTs that do not take the dynamics of the ADC into account is band limited (see, e.g., $[4,5])$. Characterization and testing of the dynamic effects of ADC's are instrumental for the performance of systems characterized by a wide bandwidth and high-dynamic range, such as contemporary and future mobile telephony systems requiring higher resolution and sampling rates.

Error tables can be used to characterize and compensate ADC's characterized by dynamic effects with short memory. Here, two types of tables that are normally considered are phase plane [6] and state space [3, 7], respectively. In a phase plane approach, the error is related to the amplitude and slope of the input, while for the state space the error is related to the current and the previous sample amplitude. In $[8,9]$, a further development of the state space method is suggested, where a generalized approach is taken with full 


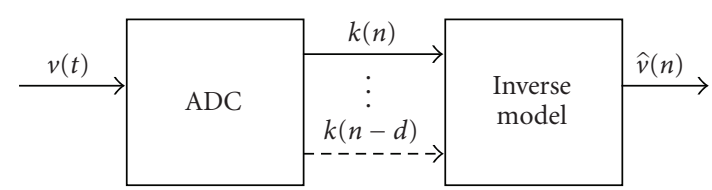

FIgURE 1: Postcorrection by using an inverse model of the ADC.

flexibility between the dynamics (i.e., the number of delayed samples) and the precision or number of bits of each sample. Thus, the size of the multidimensional lookup table is kept at a reasonable predetermined number. However, these methods are burdensome considering the time they take to train the entries of the LUT, as well as the requirement on the size of the memories. Accordingly, there is a need for dynamic postcorrection that is easy to train and simple to implement.

A parametric model requires less memory size than an LUT and does not need to be trained for every combination of present and previous samples. A well-assigned model is able to describe scenarios for which it is not trained, even though one should strive to train the model with a stimulus as realistic as possible for a given application. Different types of parametric models have been suggested in the literature, such as Volterra models and a variety of different box models (e.g., Wiener and Hammerstein models). These models describe the nonlinear dynamic behavior well, but typical error models of an ADC also include components that can not be described by nonlinear dynamic models. That implies that a postcorrection that compensate for multiple kinds of error behavior might be based on two or more models, where each error behavior is compensated with a suitable postcorrection method. The purpose of this paper is to provide a tool to evaluate to what extent a given parametric model can improve the ADC performance, when the model is used for postcorrection.

Postcorrection can be divided into two different methods. One method is to use an inverse ADC model and the other is to add a correction term. When using LUT for postcorrection, the two methods are often denoted replacement and correction, respectively. The inverse model corresponds to replacement. The output code from the ADC is a table index. The code addresses a memory, where the memory value of that address is an estimate of the analog input. The index can also be compounded by one or more previous samples (see Figure 1).

Figure 1 can also represent a correction method based on inverse models. In other words, the method is based on some mathematical system model and its inverse. Typically, a model is characterized for the ADC under test. The model gives an approximation of the input-output signal relationship. An inverse-possibly approximate- of the model is calculated, thereafter. The model inverse is used in sequence after the ADC, hence operating on the output samples, in order to reduce or even cancel the unwanted distortion.

Instead of replacing the output code from the ADC, one can add a correction term. (see Figure 2). In postcorrection using LUT, the output sample (possibly together with previous samples) addresses a correction term instead of an esti-

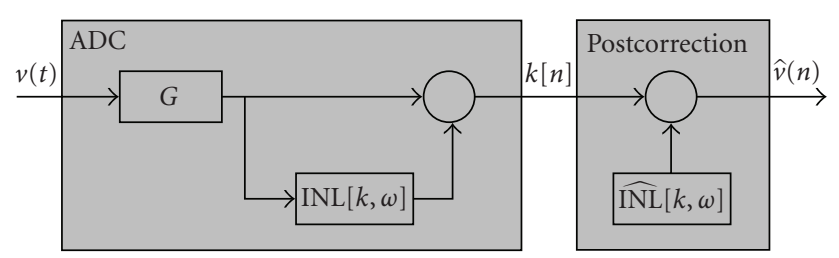

FIgURE 2: Postcorrection of an ADC by adding a correction term. The block $G$ includes analog preprocessing, sample and hold, and quantization.

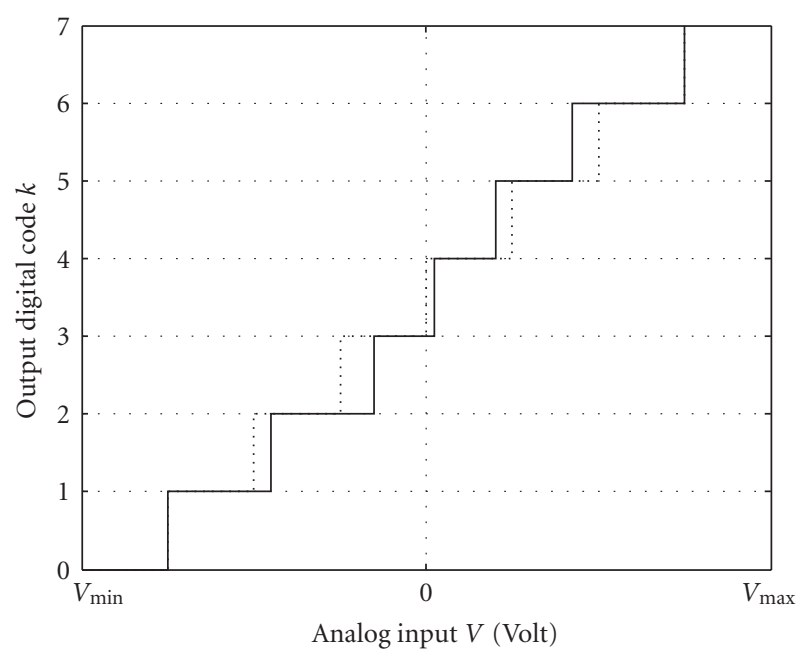

Figure 3: The relationship between the analog input signal $v$ and the digital output code $k$ from an ideal $n=3$ bits ADC (dashed line) and a practical one (solid line).

mate of the input as in the replacement method. The correction term is added to the output code. In model-based postcorrection, the postcorrection term is computed from a mathematical model. The correction term is added to the output code. In a static postcorrection, the correction term corresponds to the ADC integral nonlinearity (INL).

\section{BASIC PROPERTIES OF ADC NONLINEARITIES}

The relationship between the analog input signal $V$ [Volt] and the digital output code $k$ from an ideal ADC approximates the dotted staircase transfer curve shown in Figure 3. For the ideal ADC, the code transition levels $T_{k}$ [Volt] within the ADC range $\left(V_{\min }, V_{\max }\right)$ [Volt] are given by

$$
T_{k}=Q(k-1)+T_{1}[\text { Volt }],
$$

where $Q$ [Volt] is the ideal width of a code bin; in other words, the full-scale range of the ADC is divided by the total number of codes $\left(V_{\max }-V_{\min }\right) / 2^{N}$, where $N$ denotes the number of bits. Further, $T_{1}$ is the ideal voltage corresponding to first transition level, and $T_{1}$ is equal to $V_{\min }+Q$ or $V_{\min }+Q / 2$ depending on the convention used: the "midriser" convention or "mid-tread" convention, respectively [10]. The code $k$ spans $k=1, \ldots, 2^{N}-1$.

Due to imperfections in all practical ADC's, the transfer curve is normally somewhat distorted, which is illustrated 


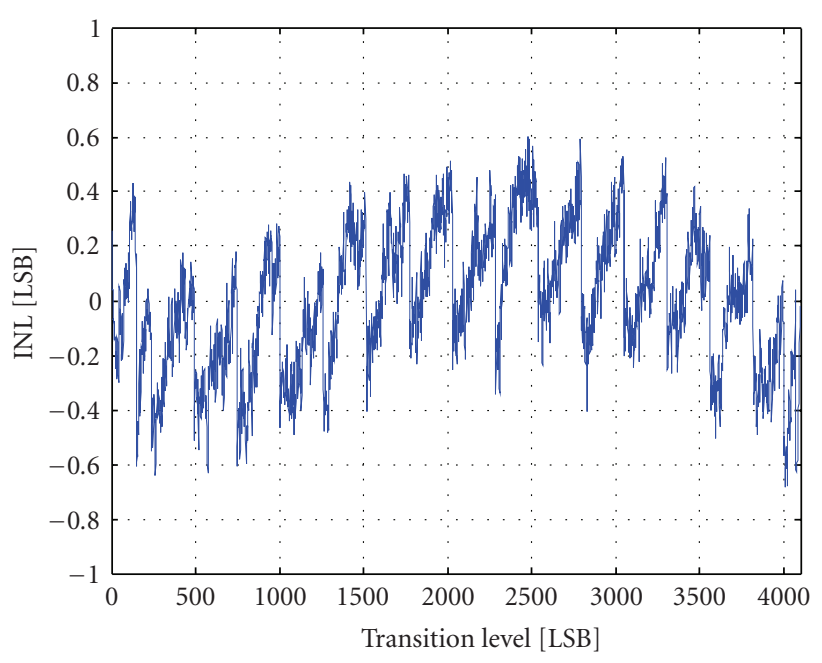

FIgURE 4: Exemplary measured INL from a 12 bit commercial ADC.

by the solid line in Figure 3. The actual code transition level $T[k]$ [Volt] (i.e., the ideal and practical transition levels are distinguished by the placement of the argument $k$, viz. $T_{k}$ and $T[k]$, resp.) is the voltage that results in a transition from ADC output code $k-1$ to $k$. The INL is described as the difference between the ideal $T_{k}$ in (1) and the actual $T[k]$ code transition levels of the ADC, after a correction has been made for gain and offset errors $[10,11]$. Given the ideal code transition levels $T_{k}$ in (1) and the measured levels $T[k]$, the correction is made by adjusting the gain $G$ and offset $V_{\text {os }}$ in order to "minimize" the residual $\varepsilon[k]$ (for $k=1, \ldots, 2^{N}-1$ ) [10],

$$
\varepsilon[k]=T_{k}-G \cdot T[k]-V_{\text {OS }}[\text { Volt }] .
$$

Equation (2) describes an overdetermined set of $2^{N}-1$ equations with the two unknowns $G$ and $V_{\text {os }}$ that are sought for. According to the IEEE standards [10], different methods may be applied for determining the optimal $\left(G, V_{\text {os }}\right)$-pair such as the "terminal-based" method [10] as used in this paper. The INL as a percentage of the full scale (FS) range of the ADC is given by the normalized residual in (2), that is,

$$
\mathrm{INL}[k]=\frac{100 \% \cdot \varepsilon[k]}{2^{N} Q}[\% \text { of FS }] .
$$

The INL is normally expressed in least significant bits (LSBs), where a LSB is synonymous with one ideal code bin width $Q$ [Volt], that is, INL $[k]=\varepsilon[k] / Q[\mathrm{LSB}]$.

Differential nonlinearity (DNL) is the difference, after correcting for the obtained static gain $G$, between a specified code bin width and the ideal code bin width $Q$, divided by the ideal code bin width. The DNL is given as follows:

$$
\operatorname{DNL}[k]=\frac{W[k]-Q}{Q}[\mathrm{LSB}],
$$

where $W[k]$ is the corrected width of code bin $k$, that is, $W[k]=G(T[k+1]-T[k])$. From (2), it follows that
$\varepsilon[k+1]-\varepsilon[k]=Q-W[k]$, and thus the relation between $\mathrm{INL}[k]$ and DNL[k] is

$$
\operatorname{DNL}[k]=\operatorname{INL}[k+1]-\operatorname{INL}[k] .
$$

The root-mean-square (RMS) value of the DNL is commonly used and given by

$$
\mathrm{DNL}_{\mathrm{RMS}}=\left(\frac{1}{2^{N-1}} \sum_{k=1}^{2^{N-1}} \mathrm{DNL}^{2}[k]\right)^{1 / 2} .
$$

\section{PARAMETRIC INL MODELING FOR ADC POSTCORRECTION}

In Figure 4, a typically measured INL from a commercial ADC is plotted. As it is evident from the plot, the behavior is a combination of a smooth wave or polynomial curve and a prickly sawtooth wave. In the following analysis, the INL will be broken up in two components; one representing the smooth curve and one representing the prickly sawtooth wave. The INL $[k]$ is then described as

$$
\mathrm{INL}[k]={ }^{\mathrm{HCF}} \mathrm{INL}^{\prime}[k]+{ }^{\mathrm{LCF}} \mathrm{INL}[k],
$$

where the first term is the contribution by the, so-called, high-code frequency component and the second term by the low-code frequency component, respectively. In [12], the static INL model was expressed as a one dimensional image in the code $k$ domain consisting of the two components. The smooth curve was entitled low-code frequency (LCF), to understand the meaning of low-frequency code, consider that the code axis represent a time axis. Accordingly, low-code frequency means slow variation over the codes, [13] component denoted by ${ }^{\mathrm{LCF}} \mathrm{INL}[k]$ and was represented by a polynomial approximation:

$$
{ }^{\mathrm{LCF}} \mathrm{INL}[k]=h_{0}+h_{1} k+h_{2} k^{2}+\cdots h_{L} k^{L}
$$

where the $h_{k}$ 's are the polynomial coefficients and $L$ is the order of the polynomial. The parameters $h_{0}$ and $h_{1}$ are typically set to zero due to the fact that INL is calculated after a correction has been made for gain and offset errors when determining the INL [10]. The high code-frequency component ${ }^{\mathrm{HCF}} \mathrm{INL}^{\prime}[k]$ is caused by a significant deviation between the polynomial approximation (8) and the actual INL $[k]$. In [14], the high-code frequency component was further divided into two parts: ${ }^{\mathrm{HCF}} \mathrm{INL}[k]$ and Noise $\mathrm{INL}[k]$, respectively. The former term, ${ }^{\mathrm{HCF}} \mathrm{INL}[k]$, depends on the physical design of the component (designs such as pipeline, successive approximation, or any other structure) and is modeled as piecewise linear $[12,15]$. The latter component, Noise $\mathrm{INL}[k]$, is the part of INL $[k]$ that cannot be described by an equation. Thus, the INL $[k]$ model in (7) is refined to

$$
\mathrm{INL}[k]={ }^{\mathrm{HCF}} \mathrm{INL}[k]+{ }^{\mathrm{LCF}} \mathrm{INL}[k]+{ }^{\text {Noise }} \mathrm{INL}[k] .
$$

A static model of an ADC and in particular the corresponding INL $[k]$ is in general not sufficient to accurately describe an ADC in a wideband application. Hence, the dynamic behavior also needs to be included in the model, which can be 
done by adding amplitude information from either previous sample amplitudes or estimates of the input slope, which is state-space and phase plane modeling, respectively. The dynamic behavior of the INL can alternatively be described as a frequency dependency, that is, different sine wave test stimuli result in different INL data. One may note that frequency selective LUTs for ADC postcorrection was considered in [16]. In order to stress the dependency of some of the components in (9) on the stimuli frequency $\omega,(9)$ is rewritten as

$$
\operatorname{INL}[k, \omega]={ }^{\mathrm{HCF}} \mathrm{INL}[k]+{ }^{\mathrm{LCF}} \mathrm{INL}[k, \omega]+{ }^{\text {Noise }} \mathrm{INL}[k, \omega],
$$

where $\omega$ denotes the normalized frequency variable,

$$
\omega=\frac{2 \pi f}{f_{s}},
$$

where $f$ is the actual frequency in Hertz and $f_{s}$ is the sampling frequency.

The main purpose of the model (10) is to use it for ADC postcorrection. The structure of the components of the model may, to some extent, be affected by the aim to find a dynamic model that is easy to train and simple to implement.

Even though the behavior models are black-box models, the arguments for having a static ${ }^{\mathrm{HCF}} \mathrm{INL}[k]$ can be justified based on some knowledge of the ADC design. The hardware structure of an ADC is consisting of two sections. First is an analog signal processing section with an amplifier and sample-and-hold circuits followed by a section performing the quantization. The high-code frequency component ${ }^{\mathrm{HCF}} \mathrm{INL}[k]$ is mainly representing the imperfections in the quantizer, which are (at least in a first approximation) static and thus depend on the code $k$ only and not on the test frequency. One favorable feature with considering the high-code frequency component to be static is that the size of the lookup table will be minimized. The low-code frequency component ${ }^{\mathrm{LCF}} \mathrm{INL}[k, \omega]$ is a two-dimensional parametric model describing a dynamic behavior. Due to the parameterization, the postcompensation can be implemented by numerical calculations.

The component ${ }^{\mathrm{LCF}} \mathrm{INL}[k, \omega]$ is described as a nonlinear dynamic model and can be described by different model structures. In [17], the input-output relation of the ADC was explored based on measured Volterra kernels [18]. In particular, in that paper it was argued for employing a constrained nonlinear Volterra model known as the parallel Hammerstein model [19]. Based on the promising results in [17], the parallel Hammerstein model is used in this work to analyze the nonlinear dynamic parts of the integral nonlinearity, as well. The basic Hammerstein model is given by a static nonlinearity, followed by a linear filter. The difference between the ordinary Hammerstein model and the parallel structure is that the contributions of different orders $\ell$ are now filtered by different filters defined by their frequency functions $\mathrm{H}_{\ell}[\omega]$, respectively. Starting with the polynomial nonlinearity $\mathrm{i}(8)$, a frequency dependency is incorporated by letting the polynomial coefficient be frequency dependent, that is,

$$
{ }^{\mathrm{LCF}} \mathrm{INL}[k, \omega]=h_{0}(\omega)+h_{1}(\omega) k+h_{2}(\omega) k^{2}+\cdots+h_{L}(\omega) k^{L} .
$$

The above dynamic nonlinearity can be described in terms of a parallel Hammerstein model with the $\ell$ th singleinput multiple-output given by $k^{\ell}$, and the zero phase linear filters with frequency function $h_{\ell}(\omega)$. In summary, the parallel Hammerstein structure with a polynomial nonlinearity is a natural generalization of the static polynomial model (8). Although in this paper constraints are imposed both on the static nonlinearity as well as the phase characteristics of the bank of linear filters, the obtained dynamic model will be used throughout this paper in order to analyze and compensate for the nonlinear dynamic parts of ADC integral nonlinearity.

The ${ }^{\mathrm{LCF}} \operatorname{INL}[k, \omega]$ is a continuous function by construction and thus models employing Volterra Kernels or a boxmodel for the transfer function are appropriate. Moreover, the noncontinuous behavior is modeled by the remaining terms, that is, ${ }^{\mathrm{HCF}} \mathrm{INL}[k]$ and ${ }^{N o i s e} \operatorname{INL}[k, \omega]$, respectively. The complete block scheme over the employed dynamic INL model is given in Figure 5. The high-code frequency component ${ }^{\mathrm{HCF}}$ INL $[k]$ depends on the code $k$ only, and not on the test frequency. Further, ${ }^{\mathrm{HCF}} \mathrm{INL}[k]$ is as in [15] assumed to be piecewise linear in the code $k$; in other words, it is described by the first-order polynomial $\alpha_{0}+\alpha_{1} k$ within a limited set of neighboring code values $k_{p}-1 \leq k<k_{p}$; denoted as the code interval $K_{p}$. The ${ }^{\mathrm{HCF}} \mathrm{INL}[k]$ is thus modeled such that

$$
{ }^{\mathrm{HCF}} \mathrm{INL}[k]=\alpha_{0}[p]+\alpha_{1}[p]\left(k-k_{p-1}\right),
$$

where $p$ refers to the ordered code interval

$$
K_{p}:\left\{k \mid k_{p-1} \leq k<k_{p}\right\}
$$

where $p=1, \ldots, P$. The initial value of $k_{0}$ is given by $k_{0}=1$, and the upper end point is, by definition, $k_{P}=2^{N}$. Typically, the number of intervals $P$ is small compared with the total number of codes, $P \ll 2^{N}-1$ (see, e.g., the INL curve given in Figure 3).

Two different postcorrection methods based on the theories given in Section 3 were presented in $[14,20]$, respectively. In both papers, the different components ${ }^{\mathrm{LCF}} \operatorname{INL}[k, \omega]$ and HCF INL $[k]$ were postcorrected separately and the dynamics were concentrated to the low-code frequency component.

\section{IDENTIFICATION OF INL MODEL PARAMETERS FROM MEASUREMENTS}

In [15], the dynamic characterization of an ADC, when using a plurality of different test frequencies in the measurement setup, was considered. In particular, the different test frequencies are denoted by the integer $m$, that is, a one-toone map to the employed set of test frequencies $f_{1} \cdots f_{M}$ in Hertz. This ordering of test frequencies attaches the notation, that is, the normalized frequency $\omega$ is below replaced by the integer $m$ corresponding to the actual test frequency $f_{m}$ (in Hertz).

The low- and high-code frequency components are parameterized and a least-square method was derived for the estimation of the parameter values from the obtained 


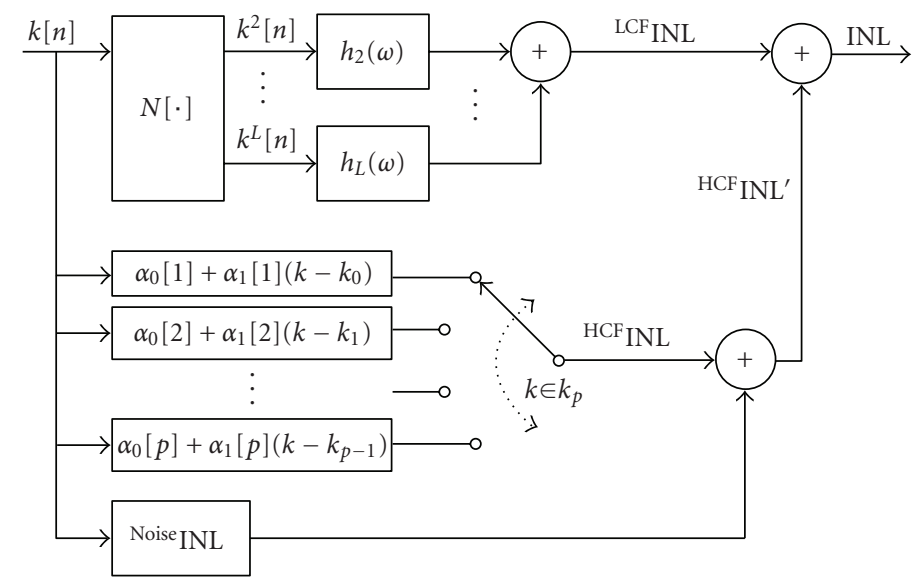

FIGURE 5: A block scheme over the complete INL model. The nonlinear block $N[\cdot]$ is a polynomial.

measurements. A closed-form solution to the estimation problem was derived. The method in [15] is reviewed below. The high-code frequency component is assumed to be piecewise linear in the code $k$, that is, described by (13) and (14). Accordingly, there are $P$ sets of polynomial coefficients $\left\{\alpha_{0}[p], \alpha_{1}[p]\right\}$, which are gathered in the parameter vector $\eta$ of size $2 P$. That is,

$$
\eta=\left(\begin{array}{c}
\alpha_{0}[1] \\
\alpha_{1}[1] \\
\vdots \\
\alpha_{0}[P] \\
\alpha_{1}[P]
\end{array}\right)
$$

The parameter vector $\eta$ in (15) describes the local gain and offset in INL for the different code intervals $K_{p}$. As previously mentioned, the high-code frequency component is, at least approximately, independent of the input test frequency. The low-code frequency component models the remaining dynamic behavior of the INL. The latter component ${ }^{\mathrm{LCF}} \mathrm{INL}[k, \omega]$ is modeled by a polynomial of order $L$ as in (12). Consider the signal model

$$
{ }^{\mathrm{LCF}} \mathrm{INL}[k, m]=f[k]^{T} \theta[m],
$$

where $T$ denotes transpose. In (16), the vector $\theta[m]=$ $\left(h_{2}[m] \cdots h_{L}[m]\right)^{T}$ is the parameter vector (i.e., $\theta[m]$ is possibly dependent on the test frequency as indicated by the argument $m$ ) and $f[k]=\left(\begin{array}{llll}k^{2} & \cdots & k^{L}\end{array}\right)^{T}$ is the regressor. One may note that each entry in the parameter vector describes the gain of the corresponding zero-phase filter in the parallel Hammerstein model. In order to estimate the parameters in the dynamic model of the integral nonlinearity, experiments are performed where in each experiment a sinewave Histogram test is used in order to pick up a set of INL data. Collecting all experimental data corresponding to the unique test frequency $f_{m}$ [Hertz] in an vector $\mathbf{y}_{m}$, a least squares fit is employed in order to fit the model parameters, so that $\mathbf{y}_{m}$ is as close as possible (in least squares) to our model, that is,

$$
\mathbf{y}_{m}=\left(\begin{array}{c}
\operatorname{INL}[1, m] \\
\vdots \\
\operatorname{INL}\left[2^{N}-1, m\right]
\end{array}\right), \quad m=1, \ldots, M
$$

In (17), $\mathbf{y}_{m}$ is the vector with experimental data and the right-hand side in our model (10), where the frequency variable used in (10) has been replaced by its corresponding integer value $m$.

We introduce (where $p$ spans $p=1, \ldots, P$ )

$$
\mathbf{g}_{p}=\left(\begin{array}{cc}
1 & 0 \\
\vdots & \vdots \\
1 & k_{p}-k_{p-1}-1
\end{array}\right)
$$

with the convention that $k_{0}=1$ and $k_{P}=2^{N}$. Further, we introduce the Vandermonde matrix $\mathbf{f}$ of size $2^{N}-1 \times L+1$ given by

$$
\mathbf{f}=\left(\begin{array}{cccc}
1^{0} & 1^{1} & \cdots & 1^{L} \\
\vdots & & & \vdots \\
\left(2^{B}-1\right)^{0} & \left(2^{B}-1\right)^{1} & \cdots & \left(2^{B}-1\right)^{L}
\end{array}\right)
$$

Then we may put the model that describes the experimental integral nonlinearity on a vector form as

$$
\mathbf{y}_{m}=\mathbf{g} \eta+\mathbf{k} \theta[m]+\mathbf{e}_{m},
$$

where $\eta$ is given by (15). Here, $\mathbf{g}$ is introduced as the block diagonal matrix with the $\mathbf{g}_{p}$ 's defined by (18) on its main diagonal, that is, $\mathbf{g}=\operatorname{blockdiag}\left(\mathbf{g}_{1}, \ldots, \mathbf{g}_{p}\right)$.

For $M$ sets of test frequencies, we have to augment the model in (20) by incorporating the multiple data sets 
$\left\{\mathbf{y}_{1}, \ldots \mathbf{y}_{M}\right\}$ and expanding the parameter vector with the $m$ dependent components, that is,

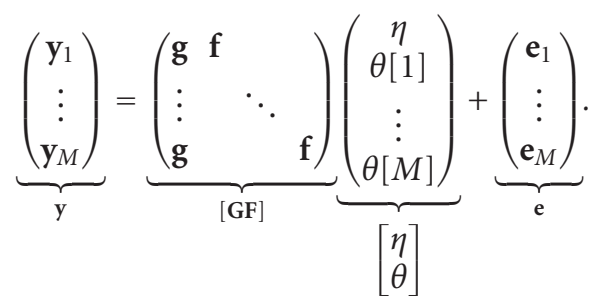

The least-squares (LS) solution of (21) is by a block matrix notation given by

$$
\left[\begin{array}{c}
\hat{\eta} \\
\hat{\theta}
\end{array}\right]=\left(\left[\begin{array}{l}
\mathbf{G}^{T} \\
\mathbf{F}^{T}
\end{array}\right]\left[\begin{array}{ll}
\mathbf{G} & \mathbf{F}
\end{array}\right]\right)^{-1}\left[\begin{array}{l}
\mathbf{G}^{T} \\
\mathbf{F}^{T}
\end{array}\right] \mathbf{y} .
$$

In [15], it is shown that the above LS solution can be separated into one solution for $\eta$ and one for each of the $M$ frequency independent parameter vectors $\theta(m)$. The complexity of the LS solution is significantly reduced by exploiting the spares structure of the involved matrices, leading to $M+1$ sets of solutions, that is,

$$
\begin{gathered}
\hat{\eta}=\left(\mathbf{g}^{T} \pi_{\perp} \mathbf{g}\right)^{-1} \mathbf{g}^{T} \pi_{\perp} \overline{\boldsymbol{y}}, \\
\hat{\theta}[m]=\left(\mathbf{f}^{T} \mathbf{f}\right)^{-1} \mathbf{f}^{T}\left(\mathbf{y}_{m}-\mathbf{g} \hat{\eta}\right), \quad m=1, \ldots, M .
\end{gathered}
$$

In (23), $\overline{\mathbf{y}}$ is defined as the average (over all test frequencies) of all INL data, that is,

$$
\overline{\mathbf{y}}=\frac{1}{M} \sum_{m=1}^{M} \mathbf{y}_{m}
$$

Further, $\pi_{\perp}$ is given by

$$
\pi_{\perp}=\mathbf{I}-\mathbf{f}\left(\mathbf{f}^{T} \mathbf{f}\right)^{-1} \mathbf{f}^{T},
$$

where I denotes the unity matrix of proper size.

We note that (23) is a linear combination of the averaged INL data (25). Regarding (24), one may note that this is the least-squares solution corresponding to the detrended data $\mathbf{y}_{m}-\mathbf{g} \hat{\eta}$.

\section{PERFORMANCE ANALYSIS}

The purpose of postcorrection is to improve the performance for the system, where the ADC's are used. Commonly used figures of merits are THD, SFDR, and SINAD. For a model-based postcorrection based on the model structure described in Section 4, and given that all model parameters are estimated, questions of relevance include the following. What are the achievable performance improvements for these figures of merits? How advanced must the postcorrection method be to meet the demands for the system?

In Section 5.1, we will formulate the relationship between a given INL model and the ADC performance, hence, the potential of improvement of an optimal postcorrection. Below, the influence of the three terms in (10) on the ADC performance measures are investigated separately. By gradually correcting for each INL term, the performance will improve step by step. We consider the case when a multiterm dynamic model of the INL (such as (10) above) is available, and is used for postcorrection as illustrated in Figure 2.

The term ${ }^{\text {Noise }}$ INL $[k, \omega]$ in (10) is throughout the paper modeled as noise, so that ${ }^{\text {Noise }} \operatorname{INL}[k, \omega]=e[k, \omega]$ is assumed to be zero mean independently identically distributed noise both in the code $k$ and in test frequency $\omega$. The general relation (10) is accordingly reduced to

$$
\operatorname{INL}[k, \omega]={ }^{\mathrm{LCF}} \operatorname{INL}[k, \omega]+{ }^{\mathrm{HCF}} \mathrm{INL}[k]+e[k, \omega] .
$$

In the following sections, we will describe the different components in more detail and how they affect the performance of the ADC. The conditions for the analysis are that the ADC performance is limited by the effects of the integral nonlinearity. In the following analysis, an expression for the THD, SINAD (and to some extent also the SFDR) as functions of the INL model parameters is developed. Consequently, the derived expressions constitute an upper limit on the performance improvements by postcorrection eliminating the effects of INL. That is, under the condition that the estimated INL used for postcorrection corresponds to the actual INL of the ADC.

\subsection{Effects of the INL low-code frequency component on ADC performance}

The ${ }^{\mathrm{LCF}} \mathrm{INL}[k, \omega]$ is a weakly nonlinear dynamic transfer function (12). Since its transfer function is a polynomial, ${ }^{\mathrm{LCF}} \mathrm{INL}[k, \omega]$ will mainly affect the harmonic distortion such as THD, and also SFDR since the 2nd and 3rd harmonics are usually the limiting spurs in SFDR. To get a measure on how the ADC performance depends on this component, an evaluation in the frequency domain will be done.

For simplicity, but without loss of generality, the analysis is performed in continuous time. Let the time domain input $v[t]$ to a static nonlinear system with output $x[t]$ be a unit amplitude cosine with zero initial phase

$$
v[t]=\cos \omega_{0} t=\frac{e^{j \omega_{0} t}+e^{-j \omega_{0} t}}{2}
$$

where $t$ is the absolute time, and $j$ is the imaginary unit $j=$ $\sqrt{-1}$. The harmonic distortion on the output $x[t]$ of a static nonlinearity driven by input $v[t]$ is given by a combination of (28) and (12), that is,

$$
\begin{aligned}
x[t]= & h_{0}+h_{1} \frac{e^{j \omega_{0} t}+e^{-j \omega_{0} t}}{2}+h_{2}\left(\frac{e^{j \omega_{0} t}+e^{-j \omega_{0} t}}{2}\right)^{2} \\
& +\cdots+h_{L}\left(\frac{e^{j \omega_{0} t}+e^{-j \omega_{0} t}}{2}\right)^{L} .
\end{aligned}
$$

Note that, we here include $h_{0}$ and $h_{1}$ since the output from the ADC is a sum of a linear quantization of the input $v[t]$ (where $h_{0}$ and $h_{1}$ are included) and the nonlinear error model given by INL $[k, \omega]$ (i.e., described by $h_{2}(\omega) \cdots h_{L}(\omega)$ according to (12)). The figures of merits used in the performance analysis below are defined for the output from the ADC. 
The even exponents produce harmonic distortion products at the ADC level and on even multiples of the fundamental frequency. Odd exponents will result in distortion at odd overtones. For example, the third-order component reads

$$
\begin{aligned}
& h_{3}\left(\frac{e^{j \omega_{0} t}+e^{-j \omega_{0} t}}{2}\right)^{3} \\
& \quad=h_{3}\left(\frac{e^{j 3 \omega_{0} t}+e^{-j 3 \omega_{0} t}+3\left(e^{j \omega_{0} t}+e^{-j \omega_{0} t}\right)}{8}\right) \\
& =h_{3}\left(\frac{1}{4} \cos 3 \omega_{0} t+\frac{3}{4} \cos \omega_{0} t\right) .
\end{aligned}
$$

In a more general case of order $n$, one can find the coefficients from the binomial theorem,

$$
(c+d)^{\ell}=\sum_{i=0}^{\ell}\left(\begin{array}{c}
\ell \\
i
\end{array}\right) c^{\ell-i} d^{i} .
$$

For example, the output $v^{\ell}[t]$ will have the following set of frequencies:

$$
v^{\ell}[t]=\left\{\begin{array}{r}
\frac{1}{2^{\ell-1}} \sum_{i=1}^{\ell}\left(\frac{1-(-1)^{i}}{2}\right)\left(\begin{array}{c}
\ell \\
\left.\frac{i-1}{2}\right) \\
\end{array}\right. \\
\frac{1}{2^{\ell-1}}\left(\frac{1}{2}\left(\begin{array}{c}
\ell \\
\frac{\ell}{2}
\end{array}\right)+\sum_{i=0}^{\ell-2}\left(\frac{1+(-1)^{i}}{2}\right)\right. \\
\left.\times\left(\begin{array}{c}
\ell \\
\frac{i}{2}
\end{array}\right) \cos \left[(\ell-i) \omega_{0} t\right]\right) \quad \ell \text { odd }
\end{array}\right.
$$

The components $v^{\ell}[t]$ are the input to the linear parts of the parallel Hammerstein model, where each branch $v^{\ell}[t]$ will be filtered by the corresponding zero-phase linear filter. The resulting output of the parallel Hammerstein model $y[t]$ reads

$$
\begin{aligned}
y[t]= & A_{0}+A_{1} \cos \left(\omega_{0} t\right) \\
& +A_{2} \cos \left(2 \omega_{0} t\right)+\cdots+A_{L} \cos \left(L \omega_{0} t\right),
\end{aligned}
$$

where the real-valued $A$-coefficients are obtained from filtering the components $v^{\ell}[t]$ given in (32) by the individual zero-phase filters in the parallel Hammerstein model

$$
\begin{gathered}
A_{0}=\frac{1}{2^{2}}\left(\begin{array}{l}
2 \\
1
\end{array}\right) h_{2}(0)+\frac{1}{2^{4}}\left(\begin{array}{l}
4 \\
2
\end{array}\right) h_{4}(0)+\cdots, \\
A_{1}=h_{1}\left(\omega_{0}\right)+\frac{1}{2^{2}}\left(\begin{array}{l}
3 \\
1
\end{array}\right) h_{3}\left(\omega_{0}\right)+\frac{1}{2^{4}}\left(\begin{array}{l}
5 \\
2
\end{array}\right) h_{5}\left(\omega_{0}\right)+\cdots, \\
A_{2}=\frac{1}{2^{1}}\left(\begin{array}{l}
2 \\
0
\end{array}\right) h_{2}\left(2 \omega_{0}\right)+\frac{1}{2^{3}}\left(\begin{array}{l}
4 \\
1
\end{array}\right) h_{4}\left(2 \omega_{0}\right)+\cdots, \\
A_{3}=\frac{1}{2^{2}}\left(\begin{array}{l}
3 \\
0
\end{array}\right) h_{3}\left(3 \omega_{0}\right)+\frac{1}{2^{4}}\left(\begin{array}{l}
5 \\
1
\end{array}\right) h_{5}\left(3 \omega_{0}\right)+\cdots
\end{gathered}
$$

For an arbitrary order $q$, one has

$$
A_{q}=\left\{\begin{array}{l}
\sum_{i=q}^{L}\left(\frac{1+(-1)^{q}}{2}\right) \frac{1}{2^{i-1}}\left(\begin{array}{c}
i \\
\frac{i-q}{2}
\end{array}\right) h_{i}\left(q \omega_{0}\right) \quad q \text { even, } \\
\sum_{i=q}^{L}\left(\frac{1-(-1)^{q}}{2}\right) \frac{1}{2^{i-1}}\left(\begin{array}{c}
i \\
\frac{i-q}{2}
\end{array}\right) h_{i}\left(q \omega_{0}\right) \quad q \text { odd. }
\end{array}\right.
$$

For a pure sinusoidal input signal, the amplitude $A_{1}$ is the fundamental tone and all $A_{q}$, where $q \neq 1$, are distortion products. Thus, the figure of merit THD (and eventually SFDR) can be expressed as a function of the amplitudes, as outlined below.

\subsubsection{Total harmonic distortion (THD)}

For a pure sinewave input of a specified amplitude and frequency, the THD is the root sum of squares (RSS) of all the harmonic distortion components including their aliases in the spectral output of the ADC. Normally, THD is estimated by the RSS of the second through the tenth harmonics, inclusive. THD is often expressed as a $\mathrm{dB}$ ratio with respect to the root mean square (RMS) amplitude of the output component at the input frequency.

The total harmonic energy (THE) for the specific subset of harmonics is defined by [10]

$$
\mathrm{THE}=\frac{1}{R^{2}} \sum_{n}\left|Y\left[\omega_{n}\right]\right|^{2},
$$

where $R$ is the length of the data record, $Y\left[\omega_{n}\right]$ is the complex value of the spectral component at frequency $\omega_{n}$. Further, $\omega_{n}$ is the $n$th harmonic frequency of the discrete Fourier transform (DFT) of the ADC output data record, $M$ is the number of samples in the data record, and $n$ is the set of harmonics over which the sum is taken. The absolute value of $Y\left[\omega_{n}\right]$ is the amplitude $A_{n}$.

Employing the above expression for the ${ }^{\mathrm{LCF}} \mathrm{INL}$, the result reads

$$
{ }^{\mathrm{LCF}} \mathrm{THE}=\frac{1}{R^{2}} \sum_{l=2}^{L}\left|Y\left[\omega_{l}\right]\right|^{2}=\frac{1}{R^{2}} \sum_{l=2}^{L} A_{l}^{2},
$$

where $L$ is the order of the polynomial in (12).

The total harmonic distortion is given by the ratio $\mathrm{THD}=\sqrt{\mathrm{THE}} / A_{1}$. The THD is often expressed as a $\mathrm{dB}$ ratio with respect to the RMS amplitude of the fundamental component of the output. The maximum achievable gain in the postcorrection of the ADC is accordingly given by the total harmonic distortion from the low-code frequency component,

$$
{ }^{\mathrm{LCF}} \mathrm{THD}=20 \log _{10}\left(\frac{\sqrt{\mathrm{LCF} T H E}}{A_{1}}\right) .
$$

\subsubsection{Spurious-free dynamic range (SFDR)}

Spurious-free dynamic range (SFDR) is the frequency domain difference in $\mathrm{dB}$ between the input signal level and the 
level of the largest spurious or harmonic component for a large, pure sinewave signal input. Including harmonics reflects common usage of the term SFDR. Normally the second or third harmonic in (36) or (37) will be the limiting spurious. Under condition that postcorrection can eliminate these harmonics, the SFDR will be limited by a nonharmonic or high-order harmonic distortion. An exact values for achievable performance it thus not possible to find.

\subsubsection{Signal to noise and distortion ratio (SINAD)}

The signal-to-noise-and distortion ratio (SINAD) is the ratio of the signal to the total noise. Unless otherwise specified, it is assumed to be the ratio of RMS signal to RMS noise, including harmonic distortion, for sinewave input signals [10]. To study the effects of ${ }^{\mathrm{LCF}}$ INL, SINAD is evaluated in the frequency domain.

Both the signal and the total noise can be determined from the DFT of data records. Let $E_{\mathrm{avm}}[\omega]$ equal the residual spectrum of $Y_{\mathrm{avm}}$ after the bins at $\omega_{m}=0$ (DC) and test frequencies, $\omega_{0}$, have all been set to zero (excised from the spectrum). Then, the RMS noise is found from the sum of all the remaining Fourier components,

$$
\text { rms noise }=\frac{1}{R}\left[\sum_{m=0}^{M-1}\left|E_{\mathrm{avm}}\left[\omega_{m}\right]\right|^{2}\right]^{1 / 2} .
$$

The contribution to the noise from ${ }^{\mathrm{LCF}}$ INL $[k]$ is ${ }^{\mathrm{LCF}} \mathrm{THE}$. The noise will thus be reduced to

$$
\text { rms noise }=\frac{1}{R}\left[\sum_{m=0}^{M-1}\left(\left|E_{\mathrm{avm}}\left[\omega_{m}\right]\right|^{2}-{ }^{\mathrm{LCF}} \mathrm{THE}\right)\right]^{1 / 2} .
$$

To conclude this section, the achievable effect from eliminating the low-code frequency component by using postcorrection is for THD given by (41) and for SINAD by (43). In indication of potential, SFDR improvement is given by (38), if the limiting spurious is an overtone.

\subsection{Effects of the high-code frequency component on ADC performance}

Since both THD and SFDR are evaluated in the frequency domain, these components can be described by a transfer function with an amplitude dependent piecewise constant amplification. How this will affect the harmonic distortion can be found from its DFT, where the error from ${ }^{\mathrm{HCF}} \mathrm{INL}[k]$ will result in a piecewise constant amplification:

$$
y_{\mathrm{HCF}}(t)=\left\{\begin{array}{cc}
\alpha_{1} \sin (\omega t)+O_{1} & \sin (\omega t)<k_{1}, \\
\alpha_{2} \sin (\omega t)+O_{2} & k_{1} \leq \sin (\omega t)<k_{2} \\
\vdots & \vdots \\
\alpha_{P} \sin (\omega t)+O_{p} & k_{P-1} \leq \sin (\omega t),
\end{array}\right.
$$

where $O$ is an offset and $\mathrm{y}_{\mathrm{HCF}}(t)$ is an virtual, internal signal in the INL model (Figure 5), namely, the ${ }^{\mathrm{HCF}}$ INL output for a single tone stimulus.
The harmonic distortion is highly dependant on the structure of ${ }^{\mathrm{HCF}} \mathrm{INL}[k]$ given in (13). Thus, one needs to solve and the DFT for (44) for the specific ADC used to get information about the effects on the THD and SFDR. However, one property of ${ }^{\mathrm{HCF}} \mathrm{INL}[k]$ worth mentioning is that the amplitudes from the harmonics from ${ }^{\mathrm{HCF}} \mathrm{INL}[k]$ are not necessarily declining with the order of the harmonic. For a smooth nonlinearity (as in the low-code frequency component) the amplitude of harmonics decreases with higher order, but this not necessarily the case for ${ }^{\mathrm{HCF}} \mathrm{INL}[k]$.

Since we are not able to find a general expression for the effect from ${ }^{\mathrm{HCF}} \mathrm{INL}[k]$ in the frequency domain, we will evaluate SINAD based on the results from [21], where the maximum achievable SINAD for a $N$-bit ADC is for a floatingpoint postcorrection given by

$$
\operatorname{SINAD}=\frac{2^{2 N}}{1+3 \cdot \mathrm{DNL}_{\mathrm{RMS}}^{2}},
$$

where $\mathrm{DNL}_{\mathrm{RMS}}$ was defined in (6). In [22], (45) was further developed to include a correction algorithm with a fixedpoint resolution for correction values.

Assume that we have eliminated the errors due to the low-code frequency component. That will give us from (27) the remaining INL $[k]$ to be ${ }^{\mathrm{HCF}} \mathrm{INL}^{\prime}[k]$,

$$
{ }^{\mathrm{HCF}} \mathrm{INL}^{\prime}[k]={ }^{\mathrm{HCF}} \mathrm{INL}[k]+e[k] .
$$

The differential nonlinearity DNL $[k]$ is found from (5), that is,

$$
\begin{aligned}
\mathrm{DNL}[k] & \\
= & { }^{\mathrm{HCF}} \mathrm{INL}^{\prime}[k+1]-{ }^{\mathrm{HCF}} \mathrm{INL}^{\prime}[k] \\
= & e[k+1]-e[k] \\
& +\left\{\begin{array}{l}
\alpha_{1}[p] \\
\alpha_{0}[p]-\alpha_{0}[p-1]-\alpha_{1}[p-1] \\
\left(k-1-k_{p-2}\right) \\
k=k_{p-1} .
\end{array}\right.
\end{aligned}
$$

Since $\{e[k]\}$ are random variables independent and identically distributed, $\mathrm{DNL}_{\mathrm{RMS}}^{2}$ yields

$$
\begin{aligned}
& \mathrm{DNL}_{\mathrm{RMS}}^{2} \\
& =\frac{1}{2^{N}-2} \sum_{k=1}^{2^{N}-2} \mathrm{DNL}^{2}[k] \\
& =\frac{1}{2^{N}-2} \sum_{k=1}^{2^{N}-2} \operatorname{Error}^{\mathrm{DNL}^{2}[k]} \\
& +\frac{1}{2^{N}-2} \sum_{p=1}^{P}\left(\left\{\alpha_{1}[p]^{2}\left(k_{p}-k_{p-1}\right)\right\}\right. \\
& \left.\quad+\left\{\alpha_{0}[p]-\alpha_{0}[p-1]-\alpha_{1}[p-1]\left(k_{p-1}-1-k_{p-2}\right)\right\}^{2}\right),
\end{aligned}
$$


where ${ }^{\text {Error }} \mathrm{DNL}[k]$ is the DNL $[k]$ related to $e[k]$. The potential for improvement from ${ }^{\mathrm{HCF}} \mathrm{INL}[k]$ is thus,

$$
\begin{aligned}
& { }^{\mathrm{HCF}} \mathrm{DNL}_{\mathrm{RMS}}^{2} \\
& =\frac{1}{2^{N}-2} \sum_{p=1}^{P}\left(\left\{\alpha_{1}[p]^{2}\left(k_{p}-k_{p-1}\right)\right\}\right. \\
& \left.\quad+\left\{\alpha_{0}[p]-\alpha_{0}[p-1]-\alpha_{1}[p-1]\left(k_{p-1}-1-k_{p-2}\right)\right\}^{2}\right) .
\end{aligned}
$$

The improvement in terms of SINAD is given by inserting (49) into (45).

\subsection{Model Errors effect on ADC performance}

The third component in (27) is considered as a zero mean independently and identically distributed noise. Thus no harmonic distortion can be expected; only nonharmonic distortion is present. The effect on THD will thus be zero, and this component affects SINAD only. The potential for SINAD improvement is [21]

$$
\mathrm{SINAD}=\frac{\left(2^{N}\right)^{2}}{\left(1+3 \cdot \text { Error }_{\mathrm{DNL}}^{2}\right)},
$$

where the root-mean-square value of DNL was introduced in (6).

In conclusion, the performance measures THD and SINAD (and to some extent SFDR) have been developed at a function of the model parameters. The objective has been to provide a tool for the user to get an estimate of what performance improvement can be achieved from an ideal postcorrection.

\section{CONCLUSIONS}

In this paper, a three-component model of an ADC aimed for postcorrection is presented. Each component has its own properties; (i) the low-code frequency component captures the dynamic component and it is modeled by a polynomial followed by linear filters, that is, a parallel Hammerstein model, (ii) the high-code frequency component is static and piecewise linear in the code $k$, and (iii) the model error is assumed to be zero mean and independent identically distributed noise.

The purpose is to provide a tool to evaluate to maximum achievable performance improvement for a modelbased postcorrection. For each of the three components, performance analyses in THD and SINAD are presented. The improvement potential for SINAD as well as the effect on THD from the low-code frequency component is given by the coefficient in the model of the ADC, while the effect from high-code frequency component on THD requires a DFT analysis.

\section{ACKNOWLEDGMENT}

This work was supported by Ericsson AB, Freescale Semiconductor Nordic AB, Infineon Technologies Nordic AB, Knowl- edge Foundation, NOTE AB, Racomna AB, Rohde\&Schwarz $\mathrm{AB}$, and Syntronic AB.

\section{REFERENCES}

[1] E. Balestrieri, P. Daponte, and S. Rapuano, "A state of the art on ADC error compensation methods," in Proceedings of the IEEE Instrumentation and Measurement Technology Conference, vol. 1, pp. 711-716, Como, Italy, May 2004.

[2] P. Arpaia, P. Daponte, and S. Rapuano, "A state of the art on ADC modelling," Computer Standards and Interfaces, vol. 26, no. 1, pp. 31-42, 2004.

[3] F. H. Irons, D. M. Hummels, and S. P. Kennedy, "Improved compensation for analog-to-digital converters," IEEE Transactions on Circuits and Systems, vol. 38, no. 8, pp. 958-961, 1991.

[4] P. Händel, M. Skoglund, and M. Pettersson, "A calibration scheme for imperfect quantizers," IEEE Transactions on Instrumentation and Measurement, vol. 49, no. 5, pp. 1063-1068, 2000.

[5] N. Björsell and P. Händel, "Histogram tests for wideband applications," IEEE Transactions on Instrumentation and Measurement, vol. 57, pp. 70-75, 2008.

[6] D. Moulin, "Real-time equalization of A/D converter nonlinearities," in Proceedings of the IEEE International Symposium on Circuits and Systems, vol. 1, pp. 262-267, Portland, Ore, USA, May 1989.

[7] J. Tsimbinos and K. V. Lever, "Improved error-table compensation of A/D converters," IEE Proceedings-Circuits, Devices and Systems, vol. 144, no. 6, pp. 343-349, 1997.

[8] H. Lundin, M. Skoglund, and P. Händel, "A criterion for optimizing bit-reduced post-correction of AD converters," IEEE Transactions on Instrumentation and Measurement, vol. 53, no. 4, pp. 1159-1166, 2004.

[9] H. Lundin, M. Skoglund, and P. Händel, "Optimal indexbit allocation for dynamic post-correction of analog-to-digital converters," IEEE Transactions on Signal Processing, vol. 53, no. 2 I, pp. 660-671, 2005.

[10] IEEE, "Std 1241-2000 IEEE Standard for Terminology and Test Methods for Analog-to-Digital Converters," 2000.

[11] T. E. Linnenbrink, J. Blair, S. Rapuano, et al., "ADC testing," IEEE Instrumentation \& Measurement Magazine, vol. 9, no. 5, pp. 37-47, 2006.

[12] L. Michaeli, P. Michalko, and J. Saliga, "Unified ADC nonlinearity error model for SAR ADC," Measurement, vol. 41, pp. 198-204, 2008.

[13] L. Michaeli, P. Michalko, and J. Saliga, "Identification of unified ADC error model by triangular testing signal," in Proceedings of the 10th Workshop on ADC Modelling and Testing, pp. 605-610, Gdynia/Jurata, Poland, July 2005.

[14] N. Björsell and P. Händel, "Dynamic behavior models of ana$\log$ to digital converters aimed for post-correction in wideband applications," in IMEKO World Congress, Rio de Janeiro, Brazil, September 2006.

[15] P. Händel, N. Björsell, and M. Jansson, "Model based dynamic characterization of analog-digital-converters at radio frequency-Invited paper," in Proceedings of the International Conference on Signal Processing and Its Applications (ISSPA '07), Sharjah, UAE, February 2007.

[16] H. Lundin, T. Andersson, M. Skoglund, and P. Händel, "Analog-to-digital converter error correction using frequency selective tables," in Proceedings of the RadioVetenskap och Kommunikation (RVK'02), pp. 487-490, Stockholm, Sweden, June 2002. 
[17] N. Björsell, P. Suchánek, P. Händel, and D. Rönnow, "Measuring Volterra kernels of analog to digital converters using a stepped three-tone scan," IEEE Transactions on Instrumentation and Measurement, pp. 1047-1050, 2006.

[18] M. Schetzen, Volterra and Wiener Theories of Nonlinear Systems, John Wiley \& Sons, New York, NY, USA, 1980.

[19] J. S. Bendat, Nonlinear Systems Techniques and Applications, John Wiley \& Sons, New York, NY, USA, 1998.

[20] L. Michaeli, L. Sochová, and J. Saliga, "ADC look-up table based post correction combined with dithering," in IMEKO World Congress, Rio de Janeiro, Brazil, September 2006.

[21] N. Giaquinto, M. Savino, and A. Trotta, "Detection, digital correction and global effect of A/D converters nonlinearities," in Proceedings of the 1st Internathional Workshop on ADC Modelling, House of Scientists, pp. 122-127, Smolenice Castle, Slovakia, May 1996.

[22] H. Lundin, P. Händel, and M. Skoglund, "Bounds on the performance of analog-to-digital converter look-up table postcorrection," to appear in Measurement. 